\title{
Alpha-particle Resonances and Alpha-clusters in Medium Light Nuclei
}

\author{
Markus Norrby* \\ Department of Natural Sciences, Åbo Akademi Universty, Turku, Finland \\ E-mail: mnorrby@abo.fi
}

\begin{abstract}
Alpha-particle resonances are of great importance in nuclear astrophysics as is well known among light nuclei. Even if the resonances occur at energies higher than what is usually available in astrophysical reactions, the resonances might make small but important contributions to reaction cross sections through the mixing of levels. The medium light nuclei ${ }^{32} \mathrm{~S},{ }^{34} \mathrm{~S}$ and ${ }^{40} \mathrm{Ca}$ have recently been investigated through elastic scattering of alpha-particles from ${ }^{28} \mathrm{Si},{ }^{30} \mathrm{Si}$ and ${ }^{36} \mathrm{Ar}$ in inverse kinematics and a very rich structure is found in the excitation functions. Data collected at several angles make it possible to find spin values for a majority of the observed resonances. These values point to a rotating cluster structure with the rotational states fragmented into many close lying levels with the same spin value.
\end{abstract}

VI European Summer School on Experimental Nuclear Astrophysics, ENAS 6

September 18-27, 2011

Acireale Italy

*Speaker. 


\section{Introduction}

The importance of nuclear resonances in nucleosynthesis was realised early on and used with great success e.g. by Hoyle et al. to explain the triple-alpha process for forming ${ }^{12} \mathrm{C}$ [1]. In light nuclei, and especially in light alpha-conjugate nuclei ${ }^{1}$, there are many known strong alpha-particle resonances at relatively low excitation energies.

Moving to nuclei heavier than ${ }^{16} \mathrm{O}$ the picture becomes more and more complicated. Among these medium light nuclei there are still clear resonances, but they are weaker and usually appear at higher excitation energies, above what is usually considered for astrophysical applications. But resent results for the nuclei ${ }^{32} \mathrm{~S},{ }^{34} \mathrm{~S}$ and ${ }^{40} \mathrm{Ca}$ presented in references $[2,3,4]$ suggest that relatively narrow resonances exist in a large excitation energy range at least for these $n \cdot \alpha$ and $n \cdot \alpha+2$ neutrons nuclei (see figure 1).

\section{Experimental methods}

When performing resonance scattering experiments there are several techniques to choose from. The simplest setup is that used by Rutherford [5] for his famous experiments where he first discovered the atomic nucleus. This method requires a very thin solid target and an ion source with variable energy. Detectors mounted at backward angles can record the angular distribution of scattered particles to find spin values for observed resonances. This method can give results with
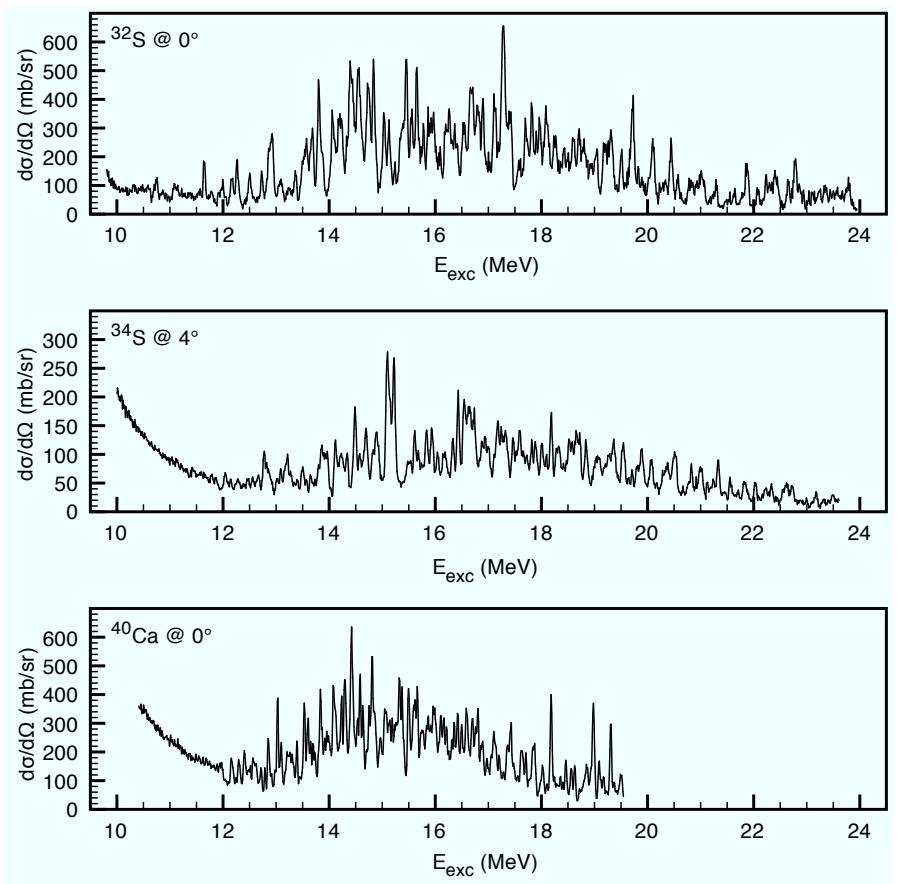

Figure 1: The excitation functions for ${ }^{32} \mathrm{~S},{ }^{34} \mathrm{~S}$ and ${ }^{40} \mathrm{Ca}$ at about $0^{\circ}$ in inverse kinematics $[2,3,4]$.

\footnotetext{
${ }^{1}$ Nuclei with possible $n \cdot \alpha$ configuration.
} 
Table 1: Comparison of some average values $[2,3,10,4])$.

\begin{tabular}{|c|c|c|c|c|}
\hline & ${ }^{32} \mathrm{~S}$ & ${ }^{34} \mathrm{~S}$ & ${ }^{36} \mathrm{Ar}^{2}$ & ${ }^{40} \mathrm{Ca}$ \\
\hline Resonances per MeV & 9.1 & 9.7 & 10.2 & 18.6 \\
Mean widths $(\mathrm{keV})$ & 69 & 56 & 104 & 31 \\
Max. cross section $(\mathrm{mb} / \mathrm{sr})$ & $\sim 600$ & $\sim 300$ & $\sim 700$ & $\sim 600$ \\
Moment of inertia $\left(\hbar^{2} / \mathrm{MeV}\right)$ & 3.9 & 2.9 & 6.8 & 3.8 \\
Average $\sum \frac{\gamma_{\alpha}^{2}}{\gamma_{s p}^{2}}(\%)^{3}$ & $<207$ & $<72$ & $<160$ & $<227$ \\
$Q_{\alpha}(\mathrm{MeV})$ & -6.9 & -7.9 & -6.6 & -7.0 \\
$S_{n}(\mathrm{MeV})$ & 15.0 & 11.4 & 15.3 & 15.6 \\
$S_{p}(\mathrm{MeV})$ & 8.86 & 10.88 & 8.51 & 8.33 \\
\hline
\end{tabular}

very good resolution, but it is very time-consuming and not practical for extensive investigations of excitation functions in large energy intervals. An example is given in reference [6] where a week of beam time was used to investigate resonances in a $1 \mathrm{MeV}$ region of the reaction $\alpha+{ }^{28} \mathrm{Si}$ with 5 $\mathrm{keV}$ steps in beam energy.

A relatively unknown method is the Thick-target backscattering technique described in [7]. In this case the setup is very similar to the example above, but instead a target thick enough to slow down the incident beam particles inside the solid material is used. With the help of simulations very good resolution in energy and angle is retained but the steps in beam energy can be much larger, in principle only limited by the energy of the first excited state of the examined nucleus. An example of the method in use is found in [8] where again the reaction $\alpha+{ }^{28} \mathrm{Si}$ was investigated.

A very effective method that has become quite popular in the last few years is the Thicktarget inverse kinematics technique (TTIK) pioneered by G.V. Goldberg et al. in Moscow [9]. For example, to examine the same $\alpha+{ }^{28} \mathrm{Si}$ reaction as above, a scattering chamber would be filled with alpha-particles (in practise helium gas) and a beam of ${ }^{28} \mathrm{Si}$ ions accelerated into the chamber as was done in [2]. If the gas is thick enough to completely stop the beam inside the camber, all possible energies and angles can be reconstructed with the help of simulations using detectors at forward angles. The method is very time-efficient and reliable, even if some resolution is sacrificed. Another advantage is that the chemical difficulties of making a solid target are avoided as long as the isotope under examination is available as a beam.

\section{Results and discussion}

The reactions $\alpha+{ }^{28} \mathrm{Si}, \alpha+{ }^{30} \mathrm{Si}$ and $\alpha+{ }^{36} \mathrm{Ar}$ were investigated in a series of experiments using a TTIK setup at the Jyväskylä University cyclotron facility in Finland [11]. The observed resonances were interpreted as highly excited states in the nuclei ${ }^{32} \mathrm{~S},{ }^{34} \mathrm{~S}$ and ${ }^{40} \mathrm{Ca}$ respectively. In connection with the analysis of these data the results from a similar experiment examining highly

\footnotetext{
${ }^{2}$ Data from the experiment were analysed only for low energies, and due to differences in experimental setup the values are not necessarily directly comparable to the values of the other isotopes.

${ }^{3}$ Known to be grossly over-estimated, but values comparable to each other.
} 

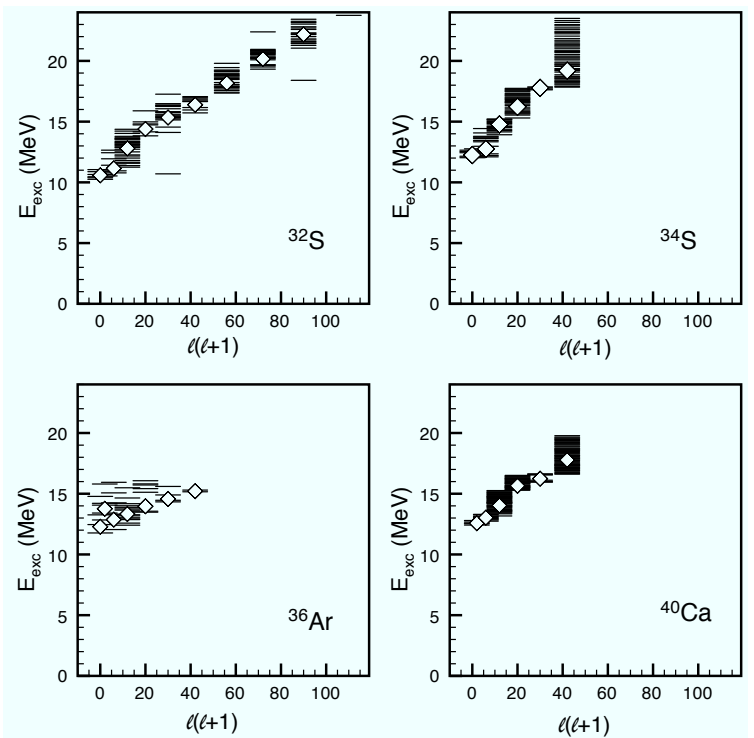

Figure 2: The $l(l+1)$ versus $E_{\text {exc }}$ plot of resonances in ${ }^{32} \mathrm{~S}$ (from [2]), ${ }^{34} \mathrm{~S}([3]),{ }^{36} \mathrm{Ar}([10])$ and ${ }^{40} \mathrm{Ca}([4])$. The diamonds represent the weighted average energy for the levels of the same spin.

excited states in ${ }^{36} \mathrm{Ar}$ were re-evaluated [10]. Some of the main results are gathered in table 1 and figure 2 .

The data show several interesting features. The very rich structure illustrated in figure 1 is confirmed by the quantitative values in table 1 . The resonances are evenly spread out over a large region in excitation energy, and the doubly magic isotope ${ }^{40} \mathrm{Ca}$ shows twice the density of resonances compared to the other nuclei. The observed resonances are very narrow, corresponding to in many cases level lifetimes several hundred times larger than the transit time of the particles involved in the reaction. The narrow resonances persist up to very high excitation energies, and seem to continue also at low energies close to the Coulomb barrier, where the resolution of the experiments quickly deteriorates. Further studies similar to [12] are needed to clarify the situation closer to the Gamow window.

Figure 2 shows a plot of spin $(l(l+1))$ and excitation energy for the resonance levels in the investigated nuclei. There is a clear linear trend indicating a quantum mechanical rotation. The very large reduced alpha-particle widths $\sum \frac{\gamma_{\alpha}^{2}}{\gamma_{s p}^{2}}$ shown in table 1 already indicate a strong alphacluster component of the highly excited levels. The rotational behaviour further contributes to a cluster interpretation of the results, and the extracted moments of inertia suggest some form of collective rotation where a number of alpha-particles orbit an inert core [13]. It is also interesting to note that, though, the cross section and reduced widths for neutron rich ${ }^{34} \mathrm{~S}$ are lower than for the $n \cdot \alpha$ nuclei, the values are still comparable and the extra neutron pair does not seem to destroy the cluster effects.

The results discussed in this paper show that resonance phenomena are very common in many nuclei. As the trend in the nuclear physics community is to concentrate basic research resources to large scale facilities examining very exotic reactions, there is a definite risk that important research on stable nuclei which could be done using relatively simple methods will be left undone. Experi- 
mental data on resonances in general and alpha-particle resonances in particular are of importance, not only for nucleosynthesis calculations, but also for developing nuclear structure theory. Hopefully the findings presented above for ${ }^{32} \mathrm{~S},{ }^{34} \mathrm{~S},{ }^{36} \mathrm{Ar}$ and ${ }^{40} \mathrm{Ca}$ will help inspire a more systematic examination of resonances and clusters in medium-light nuclei.

\section{References}

[1] F. Hoyle, On nuclear reactions occuring in very hot stars.i. the synthesis of elements from carbon to nickel, Ap. J. Supl. 11 (1954) 121.

[2] T. Lönnroth, M. Norrby, V. Z. Goldberg, G. Rogachev, M. Golovkov, K.-M. Källman, M. Lattuada, S. Perov, S. Romano, B. Skorodumov, G. Tiourin, W. Trzaska, A. Tumino, and A. Vorontsov, Highly excieted alpha-cluster states in ${ }^{32}$ studied with the thick target inverese kinematics technique, Eur. Phys. J. A 46 (2010), no. 1 5-16.

[3] M. Norrby, T. Lönnroth, V. Goldberg, G. Rogachev, M. Golovkov, K.-M. Källman, M. Lattuada, S. Perov, S. Romano, B. Skorodumov, G. Tiourin, W. Trzaska, A. Tumino, and A. Vorontsov, Highly excited alpha-cluster states in ${ }^{34}$ s, Eur. Phys. J. A 47 (2011), no. 673.

[4] M. Norrby, T. Lönnroth, V. Goldberg, G. Rogachev, M. Golovkov, K. Källman, M. Lattuada, S. Perov, S. Romano, B. Skorodumov, G. Tiourin, W. Trzaska, A. Tumino, and A. Vorontsov, Elastic alpha-particle resonances as evidence of clustering at high excitation in ${ }^{40} \mathrm{ca}$, The European Physical Journal A - Hadrons and Nuclei 47 (2011) 1-7.

[5] E. Rutherford, The scattering of alpha and beta particles by matter and the structure of the atom, Philos. Mag. (Series 6) 21 (1912).

[6] M. Brenner, N. R. Fletcher, J. A. Liendo, S. E. Belov, D. D. Caussyn, T. K. Nieto, and S. H. Myers, Resonances above 14 mev in alpha-particle scattering from silicon, Acta Phys. Hung. N.S. 11 (2000) 221.

[7] K.-M. Källman, V. Goldberg, T. Lönnroth, P. Manngård, A. Pakhomov, and V. Pankratov, Thick-target backscattering technique to measure alpha-particle elastic scattering cross sections, Nucl. Instr. Meth. Phys. Res. A338 (1994) 413-418.

[8] K.-M. Källman, M. Brenner, V. Goldberg, T. Lönnroth, P. Manngård, A. Pakhomov, and V. Pankratov, Narrow alpha +28 si elastic-scattering states at high excitation in 32s, Eur. Phys. J. A 16 (2003) 159-169.

[9] V. Z. Goldberg and A. E. Pakhomov, Possibility of generating radioactive beams by means of a cyclotron and a magnetic separator for an inverse-kinematics study of resonance elastic scattering, Phys. At. Nucl. 56 (1993) 1167.

[10] V. Z. Goldberg, G. V. Rogachev, M. Brenner, K. M. Källman, T. Lönnroth, M. V. Rozhkov, S. Torilov, W. H. Trzaska, and R. Wolski, Observation of an alpha-cluster structure in 36ar, Physics of Atomic Nuclei 63 (2000), no. 9 1518-1526.

[11] E. Liukkonen, New Cyclotron at Jyväskylä, p. 22. Proceedings of the 13th International Conference on Cyclotrons and their Applications: Vancouver, BC, Canada, 6-10 July 1992. World Scientific Publishing Co., Singapore, 1992.

[12] K.-M. Källman, Low energy scattering of a-particles by 28si, Z. Phys. A 356 (1996), no. 3 287-291.

[13] M. Norrby and T. Lönnroth, Evidence of rotational behavior of alpha-clusters in 32s, 34s and 40ca, Int. J. Mod. Phys. E 20 (2011), no. 41042. 